\title{
Impact of Charge Correlation, Chain Rigidity and Chemical Specific Interactions on the Behavior of Weak Polyelectrolytes in Solution
}

\author{
Andrea Tagliabue,$^{\dagger}$ Lorella Izzo, $^{\dagger}$ and Massimo Mella*, ${ }^{\dagger}$ \\ Dipartimento di Scienza ed Alta Tecnologia, Università degli Studi dell'Insubria, via \\ Valleggio 9, 22100, Como, Italy, and Dipartimento di Biotecnologie e Scienze della Vita, \\ Università degli Studi dell'Insubria, via J. H. Dunant 3, 21100, Varese, Italy \\ E-mail: massimo.mella@uninsubria.it
}

${ }^{*}$ To whom correspondence should be addressed

†Dipartimento di Scienza ed Alta Tecnologia, Università degli Studi dell’Insubria, via Valleggio 9, 22100, Como, Italy

${ }^{\ddagger}$ Dipartimento di Biotecnologie e Scienze della Vita, Università degli Studi dell’Insubria, via J. H. Dunant 3, 21100, Varese, Italy 


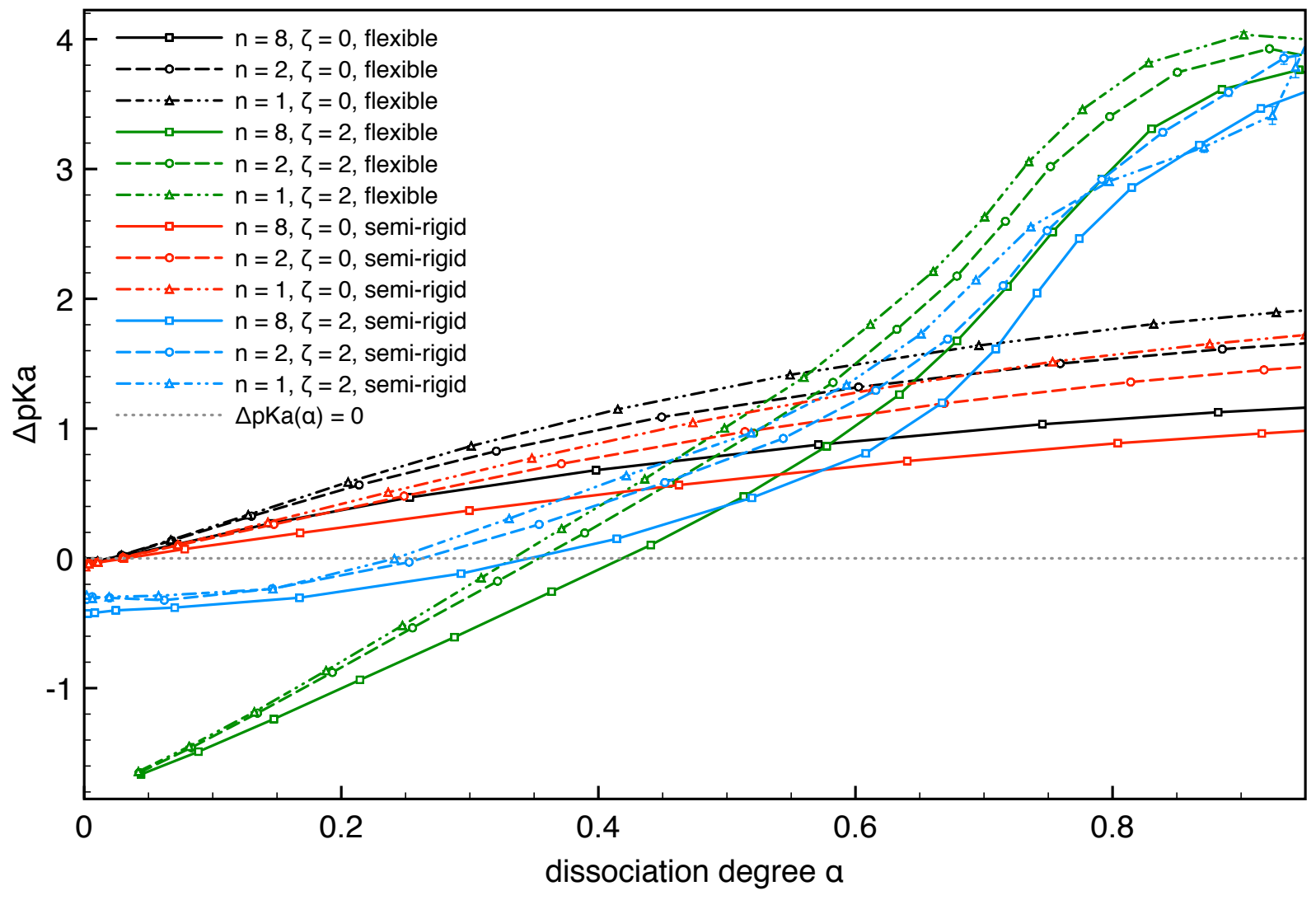

Figure S1: $\Delta \mathrm{p} K_{a}$ as function of $\alpha$ for different number of chains in the cell, precisely for $n=8$ (solid lines, squares), $n=2$ (dashed lines, circles) and $n=1$ (dotted-dashed lines, triangles). Since the number of monomers for each chain is $L=15, n=1,2,8$ correspond to values of chains concentration equal to $C \simeq 1.37 \cdot 10^{-3} \mathrm{M}, 2.74 \cdot 10^{-3} \mathrm{M}, 1.10 \cdot 10^{-2} \mathrm{M}$, and, since $L=15$, to values of monomers concentration equal to $C_{\mathrm{m}} \simeq 2.06 \cdot 10^{-2} \mathrm{M}, 4.11 \cdot 10^{-2} \mathrm{M}$, and $1.65 \cdot 10^{-1} \mathrm{M}$, respectively. The following cases are presented: flexible chains with $\left(\zeta=2, k_{\text {bend }}=0\right.$, green) and without $(\zeta=0$, $k_{\text {bend }}=0$, black $)$ MB interactions, and semi-rigid chains with $\left(\zeta=2, k_{\text {bend }}=2 \cdot 10^{-3} k_{B} T / \operatorname{deg}^{2}\right.$, red) and without $\left(\zeta=0, k_{\text {bend }}=2 \cdot 10^{-3} k_{B} T / \mathrm{deg}^{2}\right.$, light blue) the possibility to form c-H-bonds. The dotted gray line is a guide for the eye useful to discern negative and positive values of $\Delta \mathrm{p} K_{a}$. Standard error bars are smaller than plot symbols when not visible. 


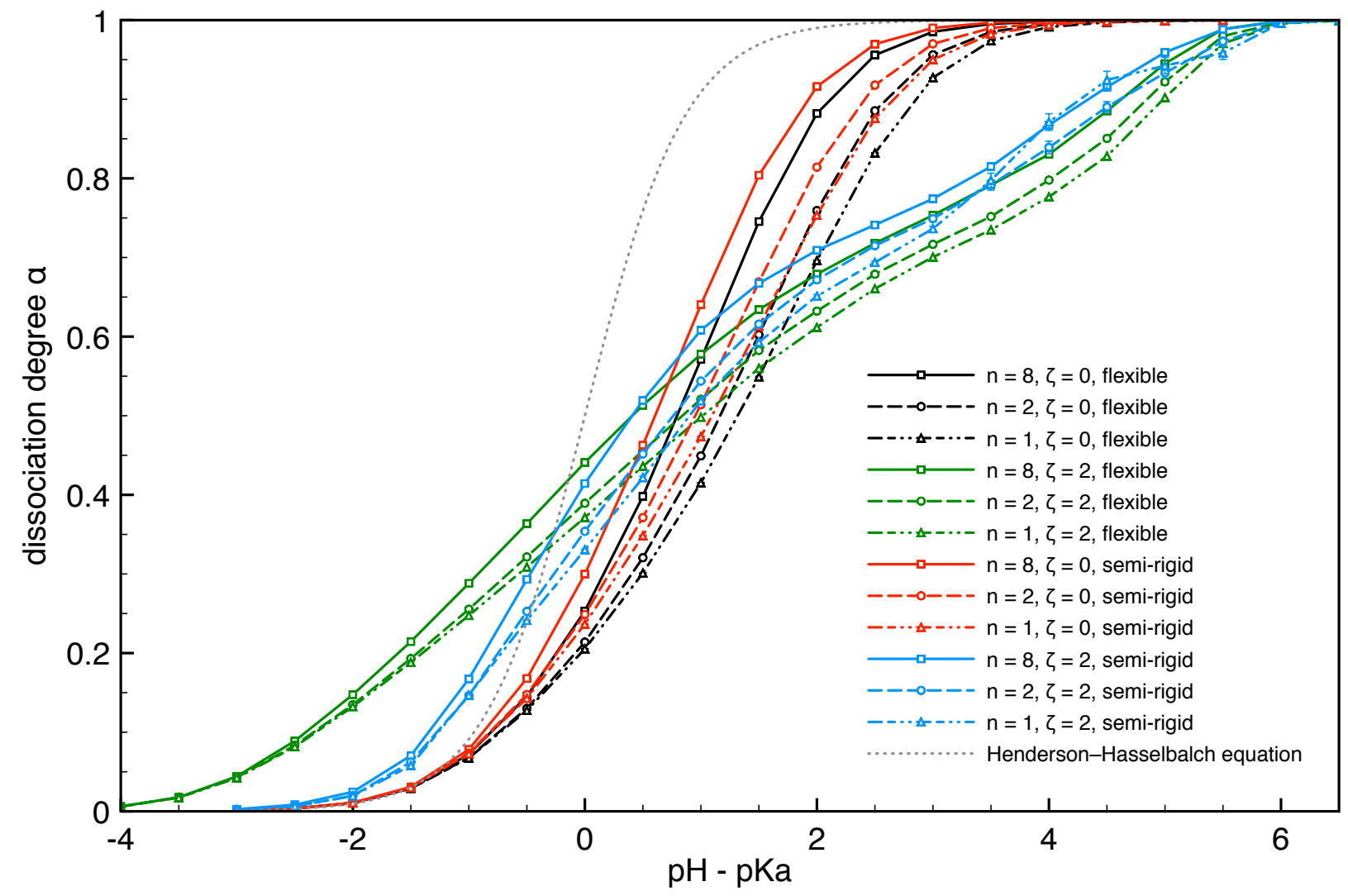

Figure S2: Dissociation degree $\alpha$ as function of the control parameter $\mathrm{pH}-\mathrm{p} K_{a}$ for the same cases presented in Figure S1 (the color scheme is maintained). Here, the dotted gray line indicates the ideal behavior predicted by the Henderson-Hasselbalch equation. Standard error bars are smaller than plot symbols when not visible. 

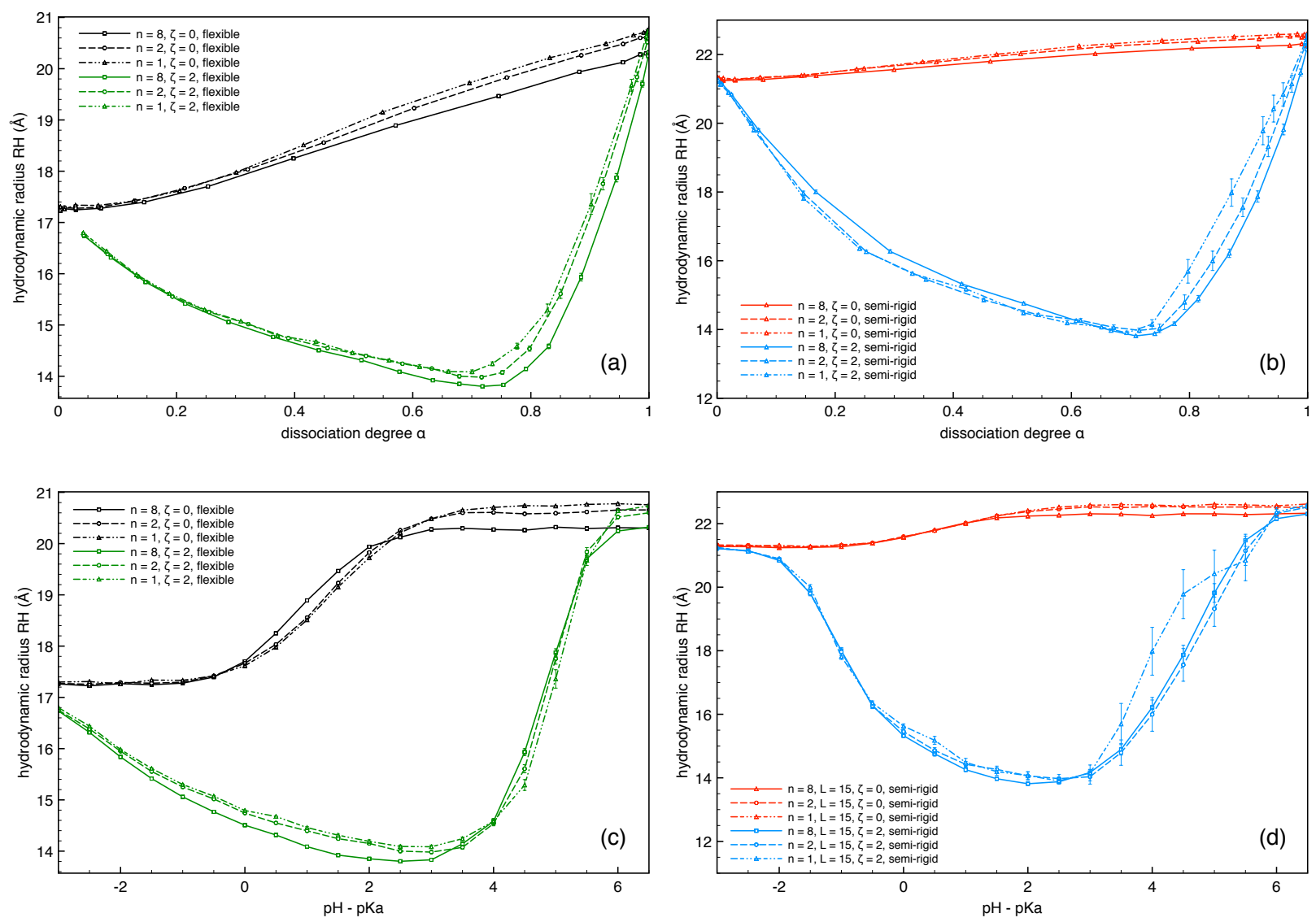

Figure S3: Hydrodynamic radius $R_{H}$ as function of $\alpha$ (top panels) or $\mathrm{pH}-\mathrm{p} K_{a}$ (bottom panels) for the same cases presented in Figure S1 (the color scheme is maintained). 

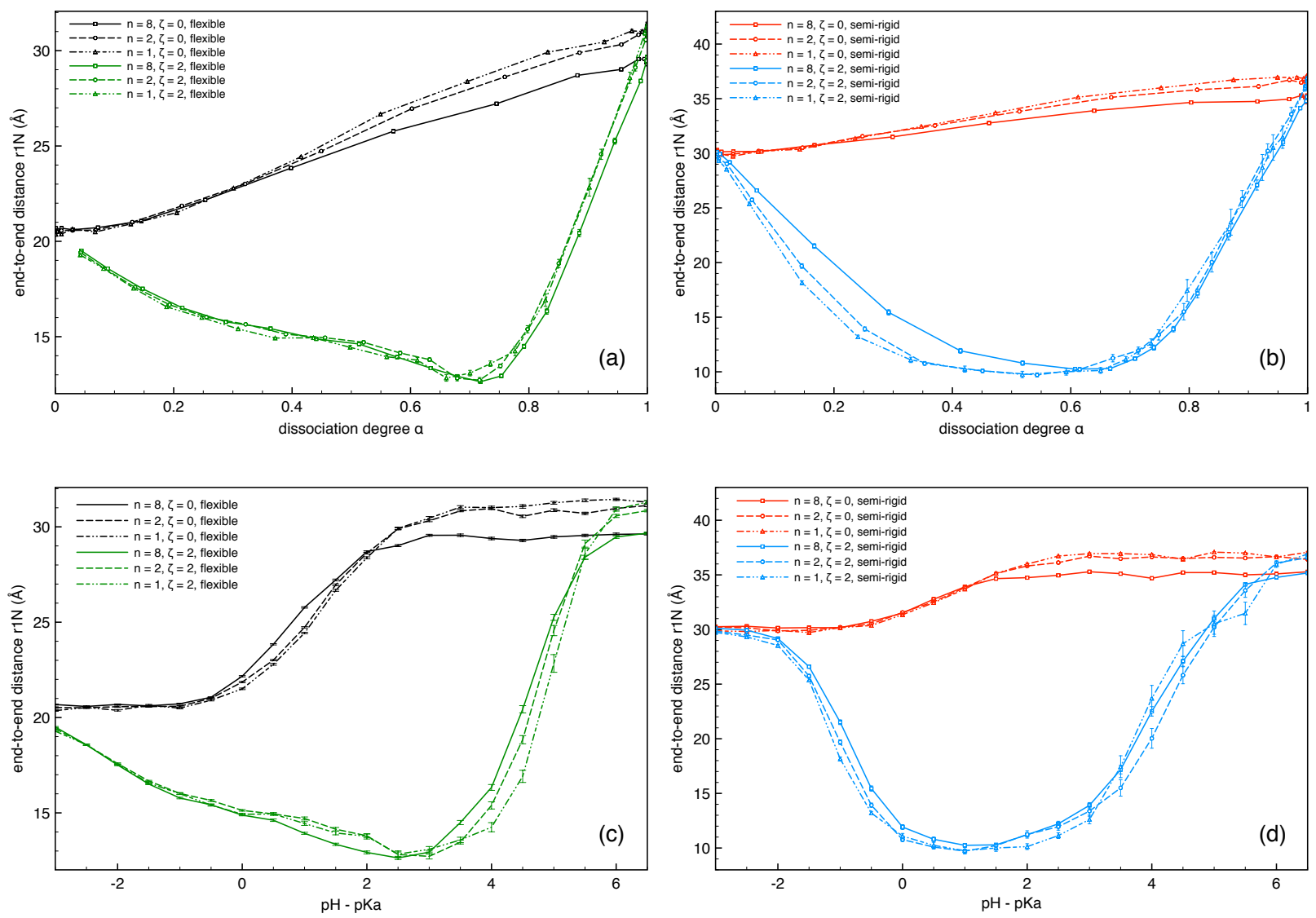

Figure S4: End-to-end distance $r_{1 \mathrm{~N}}$ as function of $\alpha$ and $\mathrm{pH}-\mathrm{p} K_{a}$. The scheme is the same as in Figure S3. 

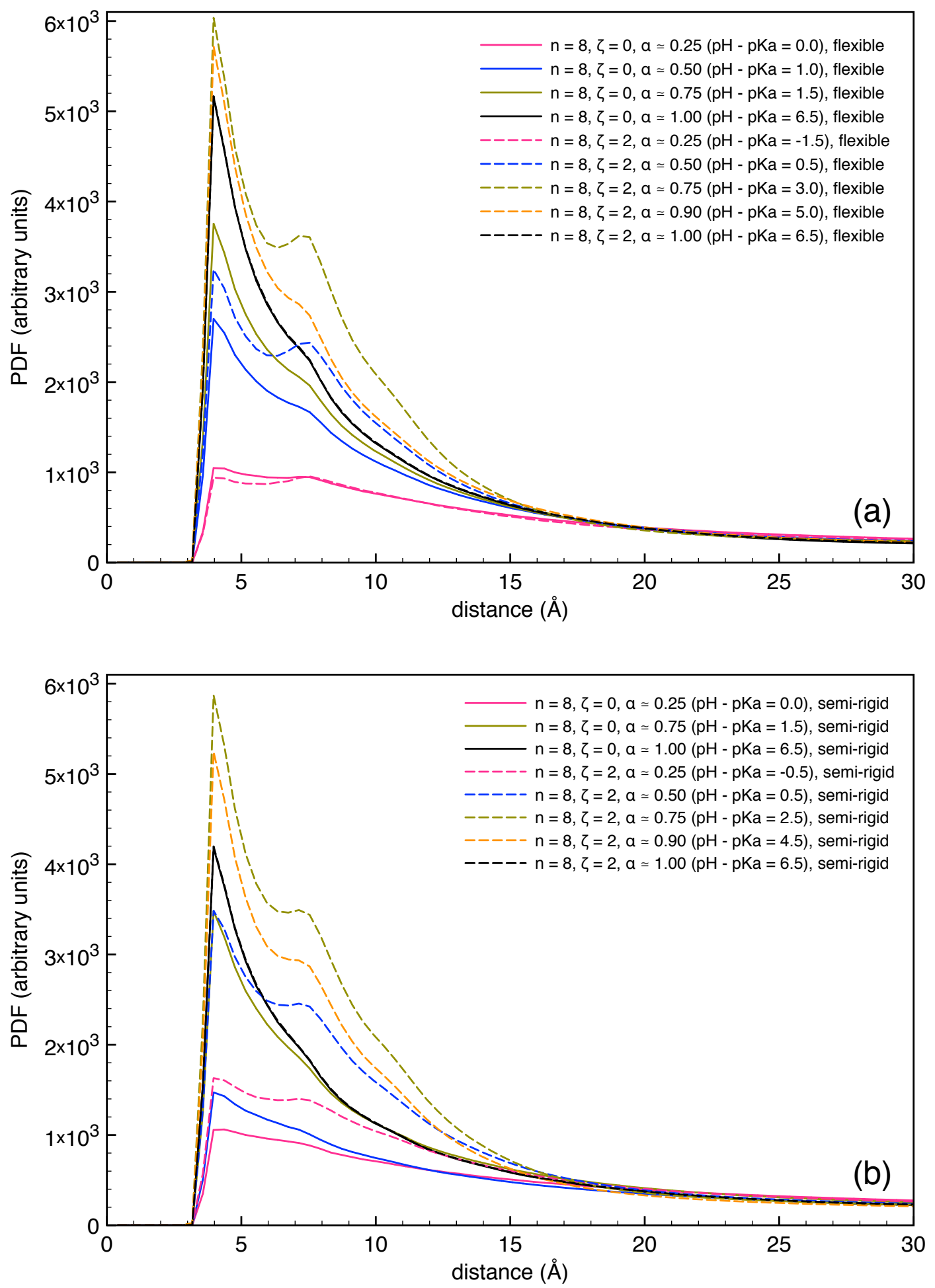

Figure S5: Pair distribution functions calculated between monomers (both neutral and charged) and counterions, with (dashed lines) and without (solid lines) MB interactions. Shown are data for different dissociation degree values: $\alpha \simeq 0.25$ (pink), $\alpha \simeq 0.50$ (blue), $\alpha \simeq 0.75$ (olive green), $\alpha \simeq 0.90$ (orange), $\alpha \simeq 1.00$ (black). (a) flexible chains; (b) semi-rigid chains. Each distribution value has been divided by the corespondent volume element. 

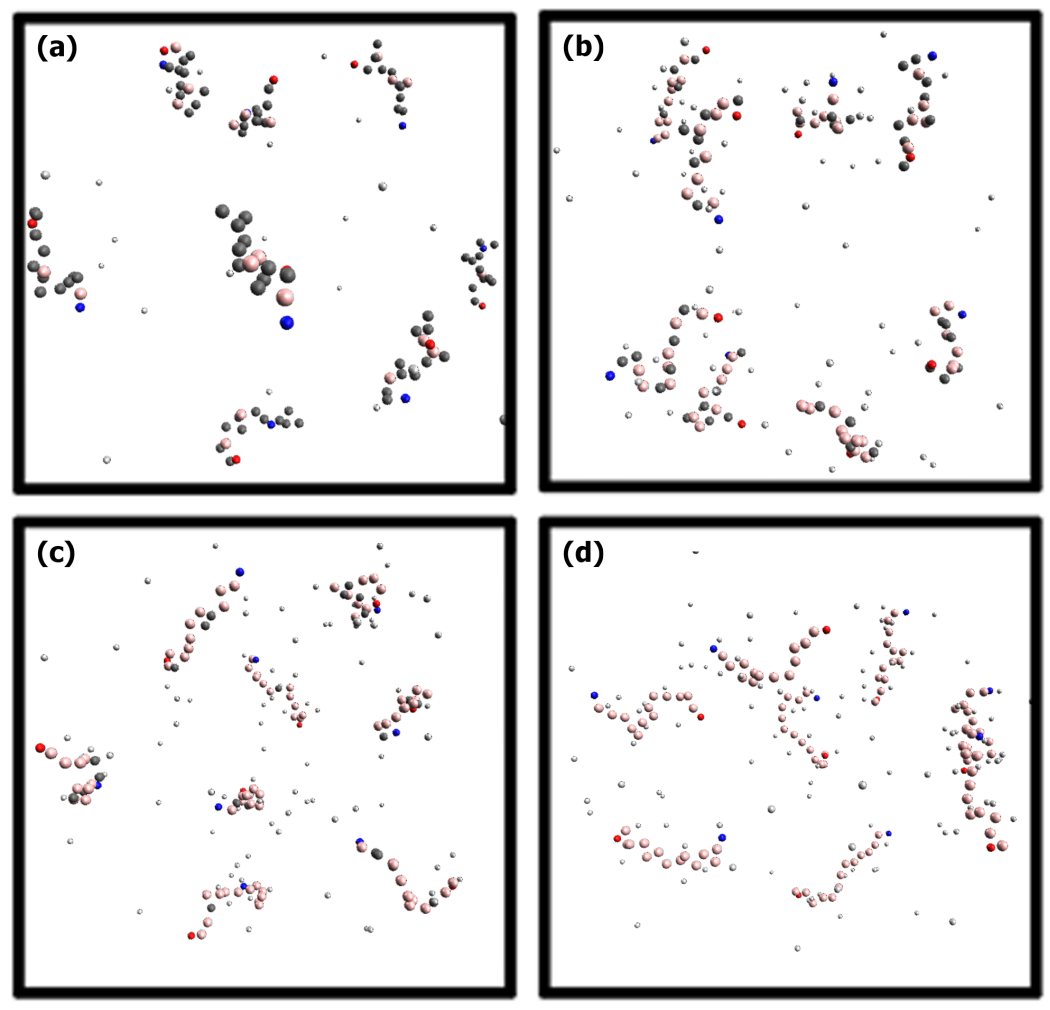

Figure S6: Trajectory snapshots for $n=8$ flexible chains and $\zeta=0$ interactions with different dissociation degrees: (a) $\alpha=0.25$, (b) $\alpha=0.50$, (c) $\alpha=0.75$, (d) $\alpha=1.00$. Color scheme: neutral monomers in dark gray, charged monomers in pink, counterions in white; the first and the last monomer of each chain are depicted in red and blue, respectively. 

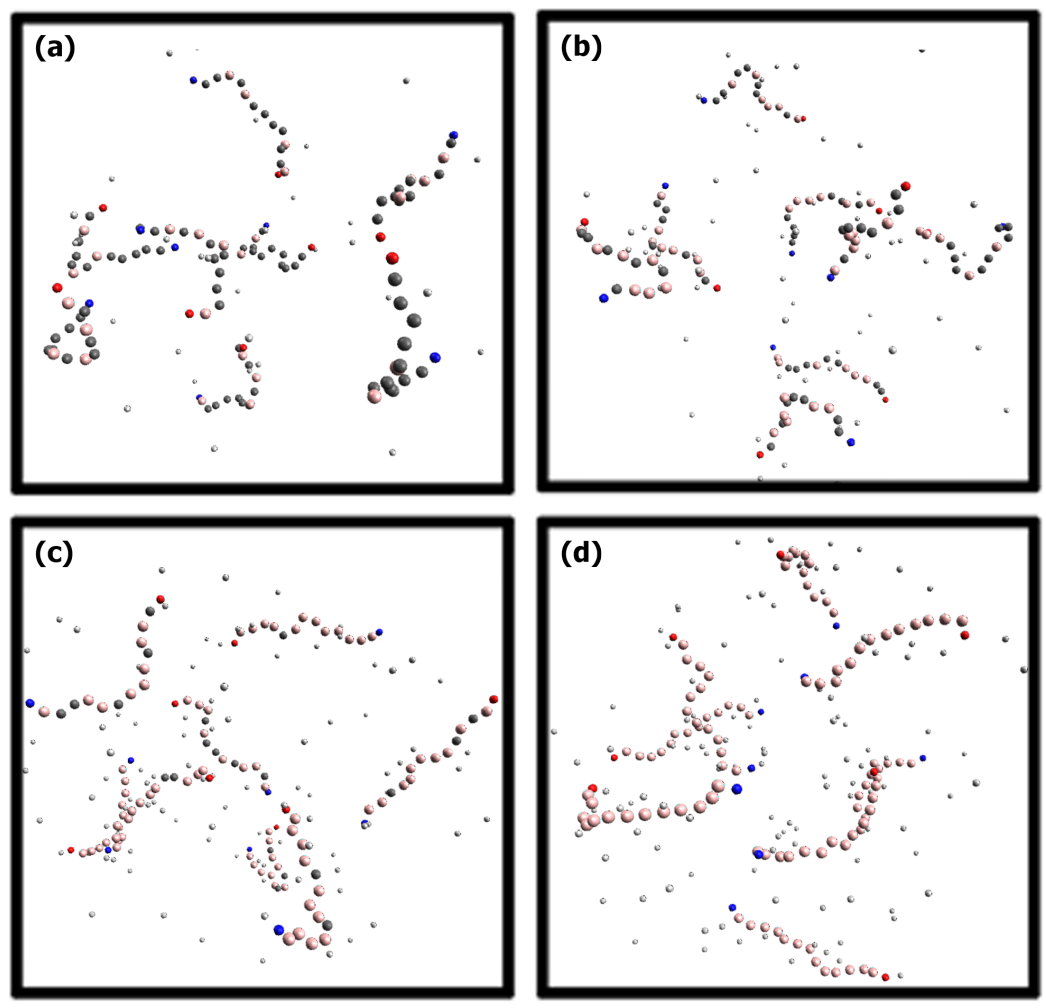

Figure S7: Trajectory snapshots for $n=8$ semi-rigid chains and $\zeta=0$ with different dissociation degrees: (a) $\alpha=0.25$, (b) $\alpha=0.50$, (c) $\alpha=0.75$, (d) $\alpha=1.00$. Color scheme as in Figure S6. 

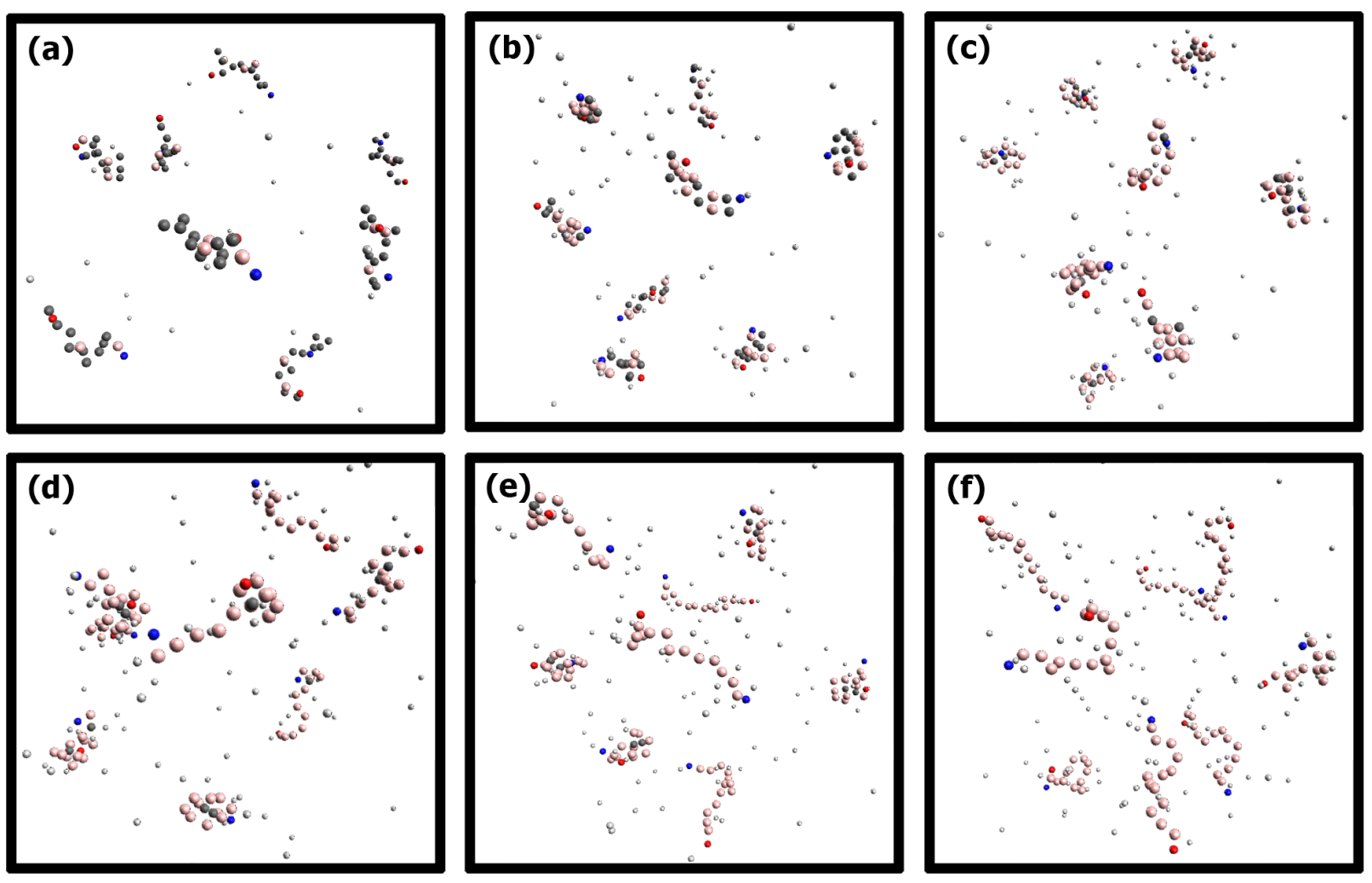

Figure S8: Trajectory snapshots for $n=8$ flexible chains and $\zeta=2$ for different dissociation degrees: (a) $\alpha=0.25$, (b) $\alpha=0.50$, (c) $\alpha=0.75$, (d) $\alpha=0.80$, (e) $\alpha=0.90$, (f) $\alpha=1.00$. Color scheme as in Figure S6. 

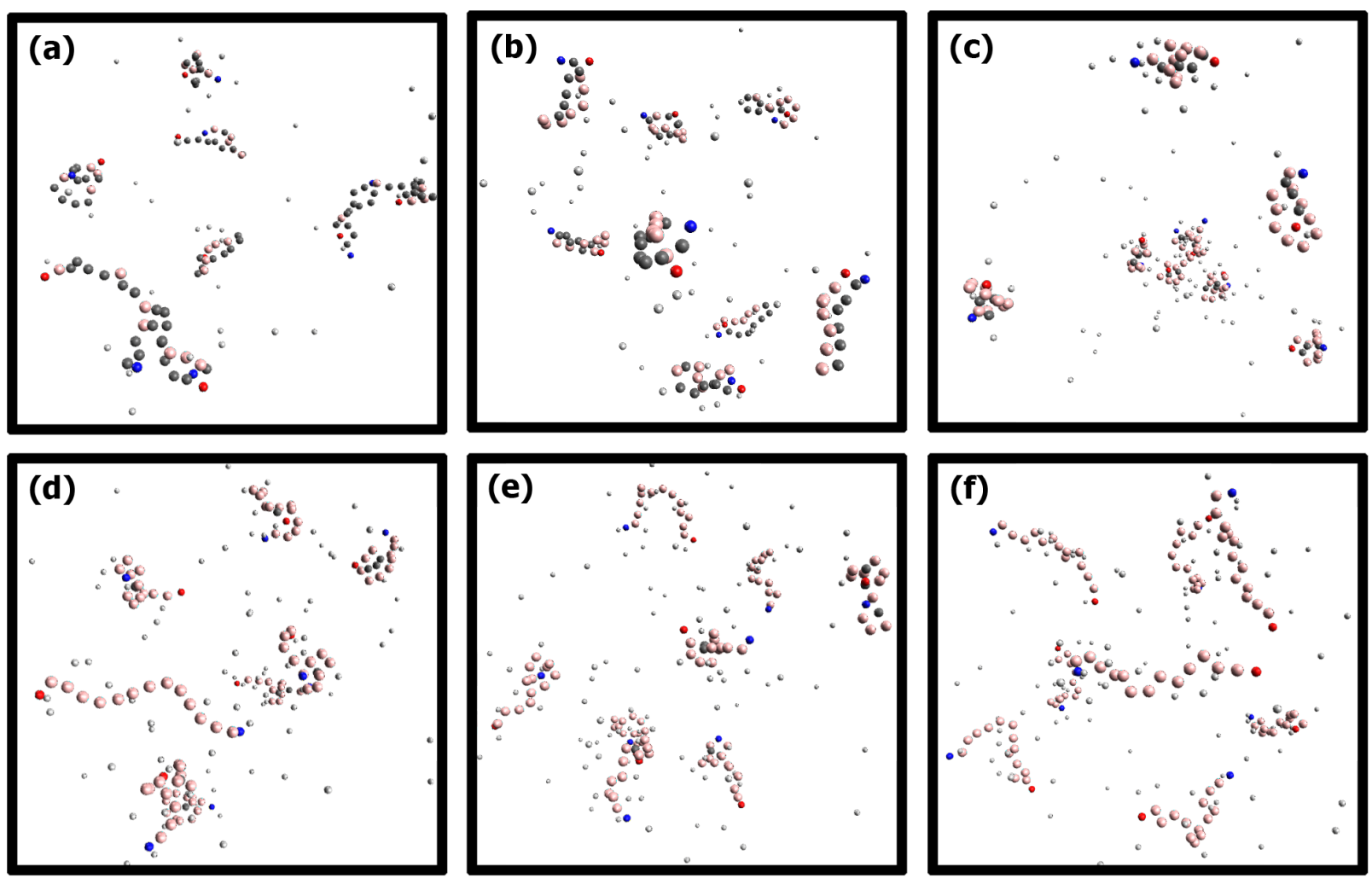

Figure S9: Trajectory snapshots for $n=8$ semi-rigid chains and $\zeta=2$ for different dissociation degrees: (a) $\alpha=0.25$, (b) $\alpha=0.50$, (c) $\alpha=0.75$, (d) $\alpha=0.80$, (e) $\alpha=0.90$, (f) $\alpha=1.00$. Color scheme as in Figure S6. 

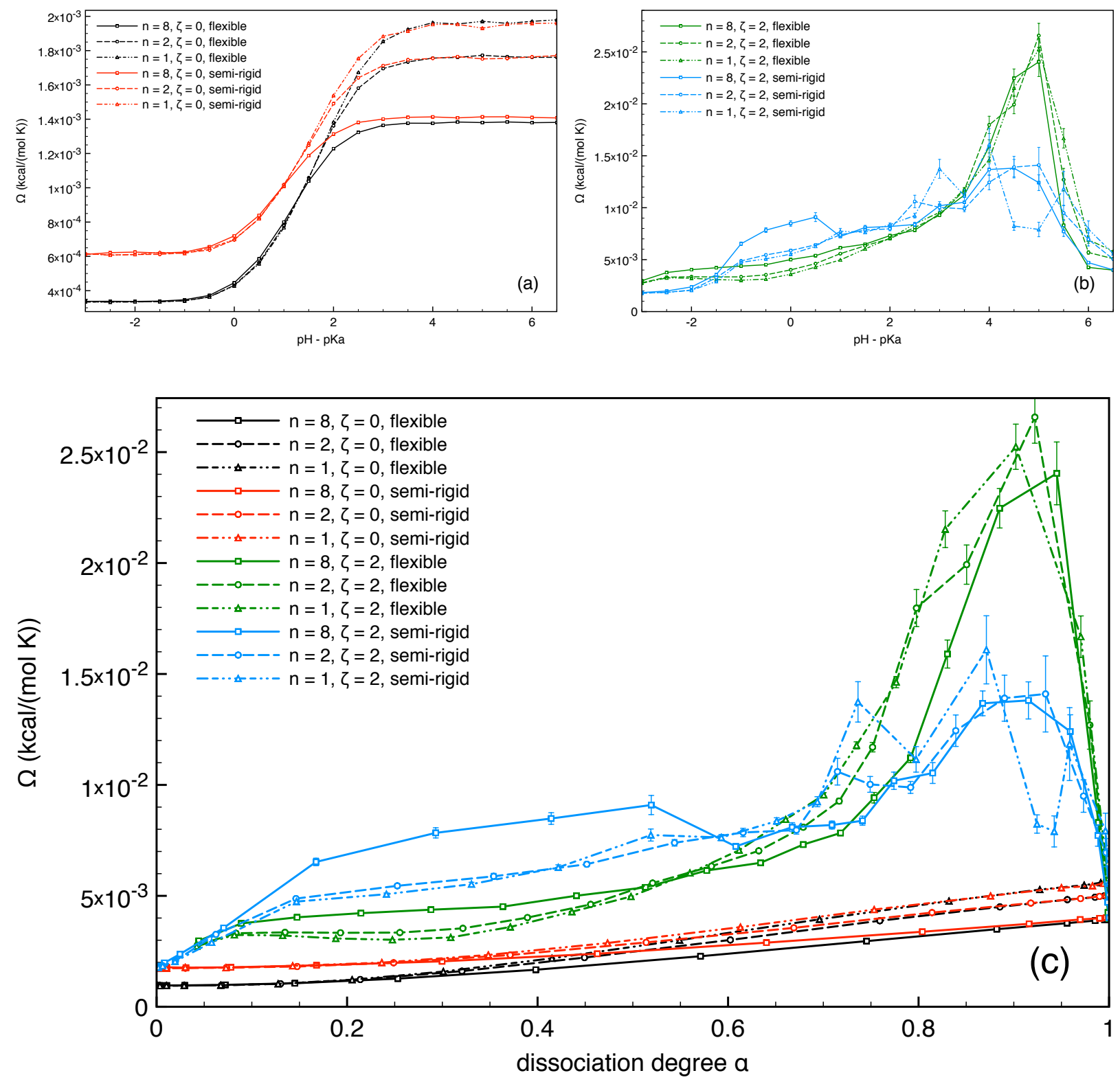

Figure S10: $\Omega$ in $\mathrm{kcal} /(\mathrm{mol} \mathrm{K})$ as versus: (a), (b) $\mathrm{pH}-\mathrm{p} K_{a}$; (c) $\alpha$. 


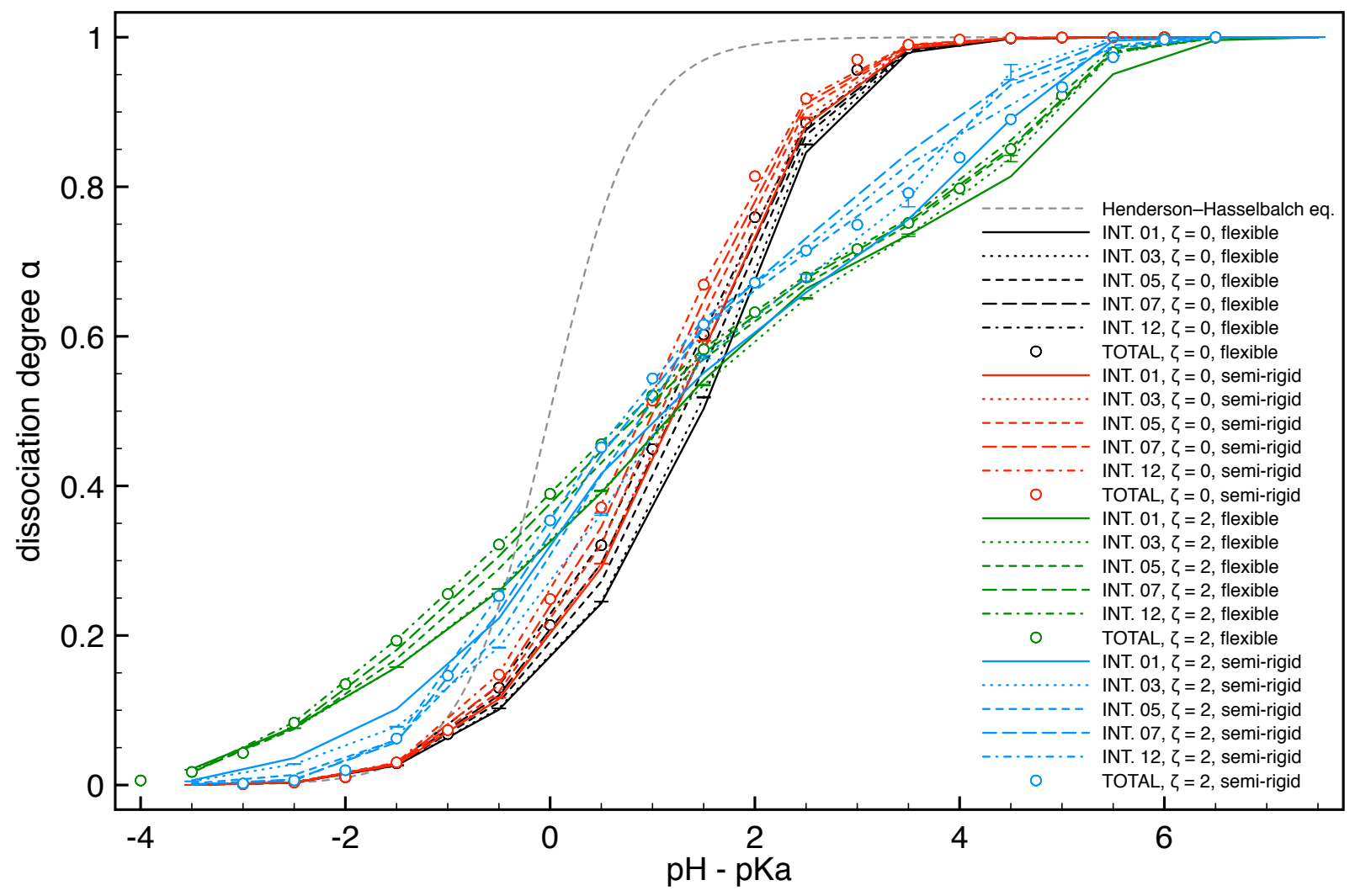

Figure S11: Dissociation degree $\alpha$ as function of the control parameter $\mathrm{pH}-\mathrm{p} K_{a}$ calculated for different $d_{\text {CoMs }}$ intervals. The color scheme is the same as in Figure S1. Here, the dashed gray line indicates the ideal behavior predicted by the Henderson-Hasselbalch equation. In order to make the chart more readable, error bars has been plotted only for the "INT 03" case. 

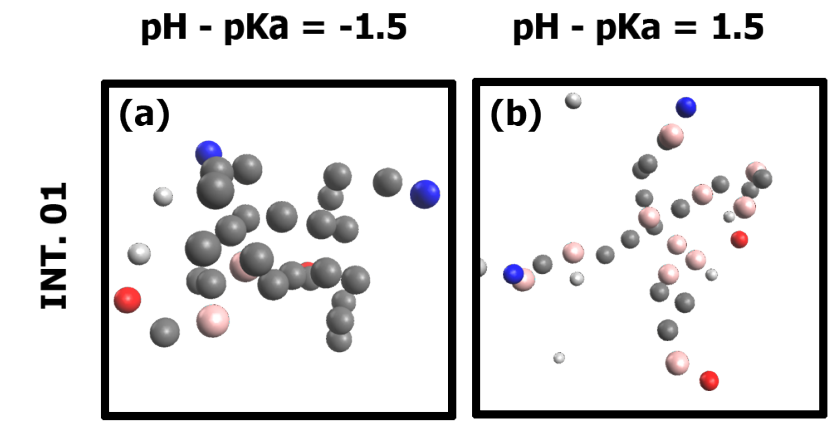

pH - pKa $=2.5$

$\mathrm{pH}-\mathrm{pKa}=4.5$
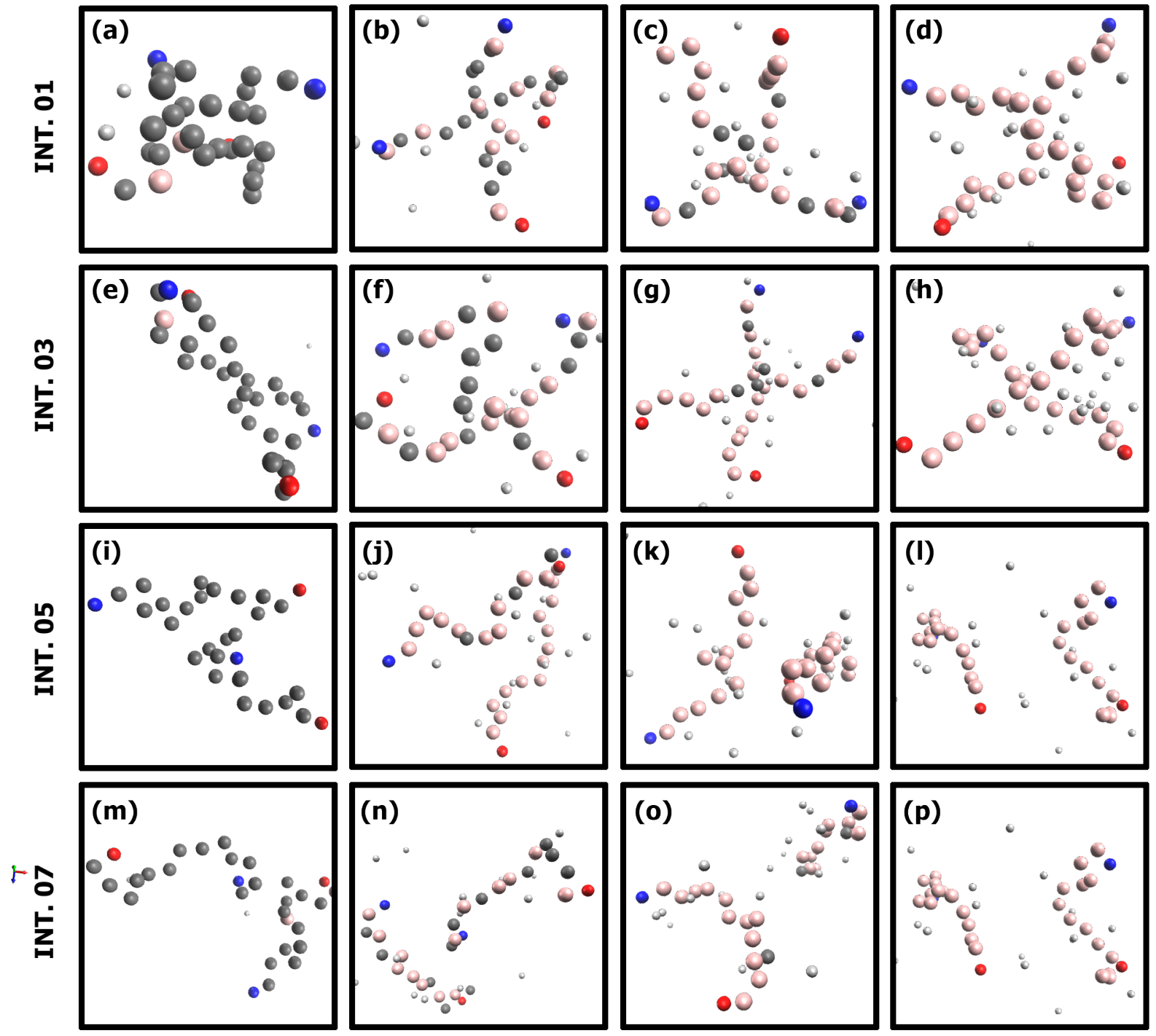

Figure S12: Trajectory snapshots for 2 flexible chains and $\zeta=0$ at different $\mathrm{pH}-\mathrm{p} K_{a}$ values and $d_{\text {CoMs }}$ distances. Color scheme as in Figure 6. 


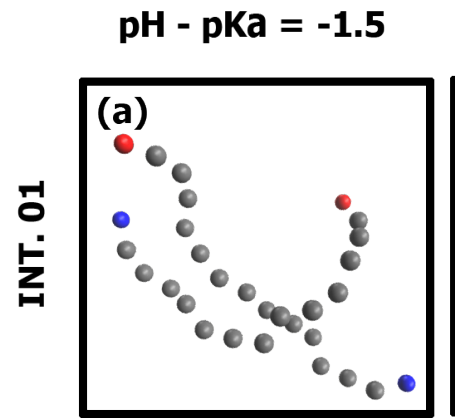

pH - pKa $=1.5$
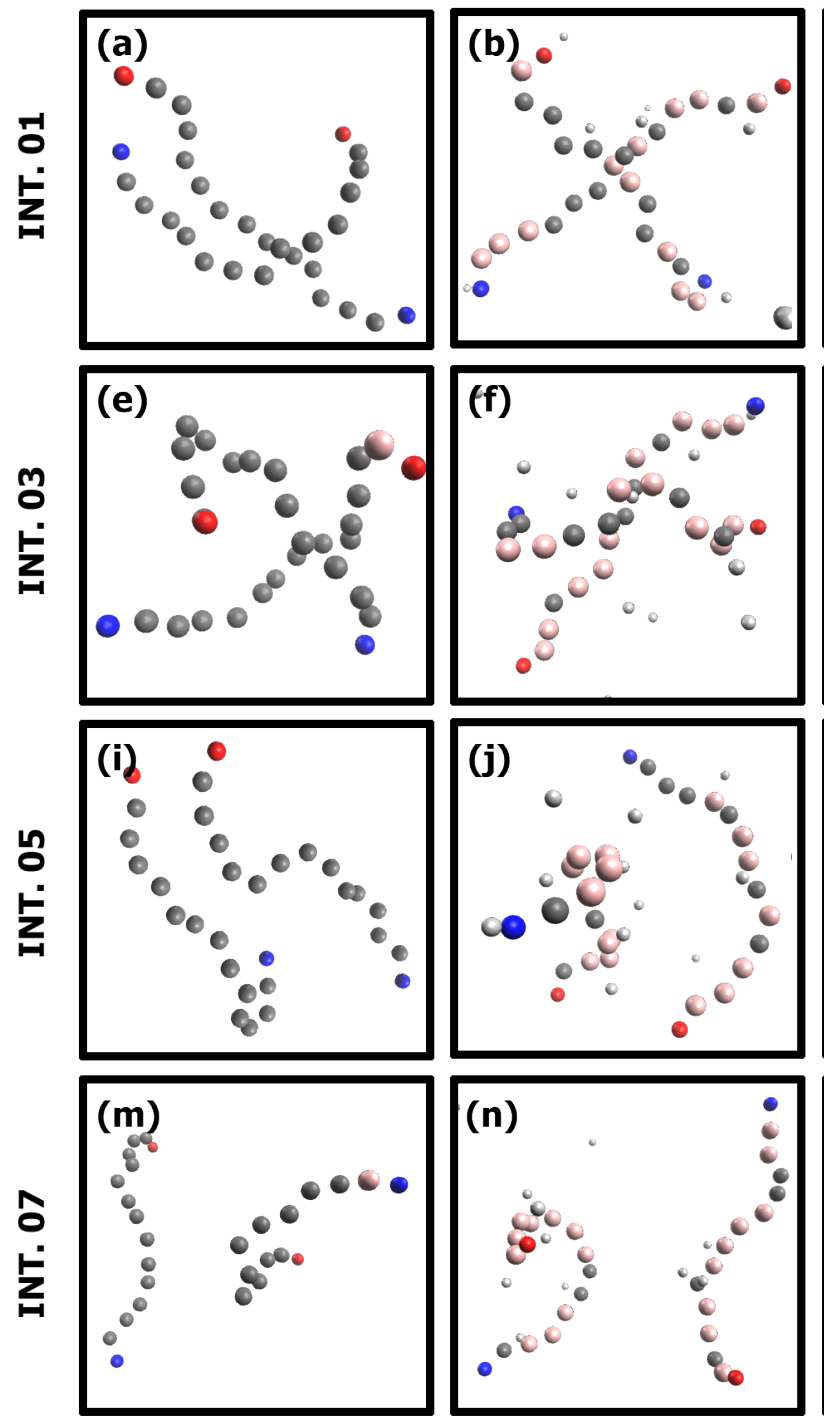
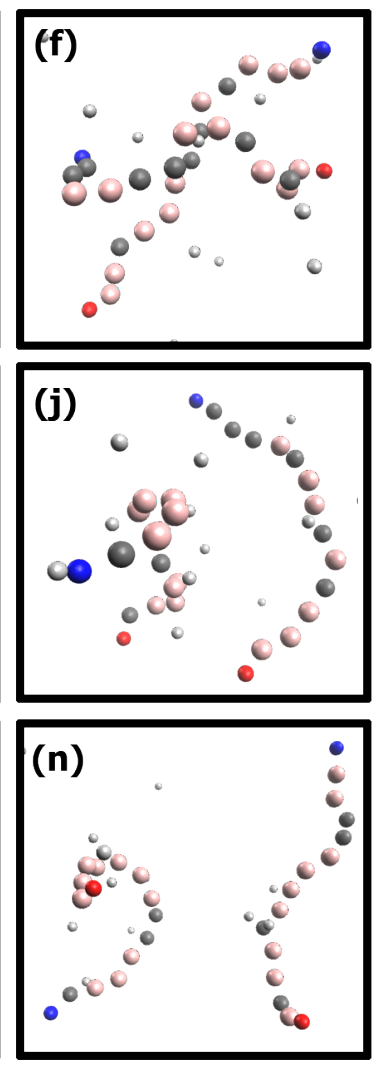

pH - pKa $=2.5$
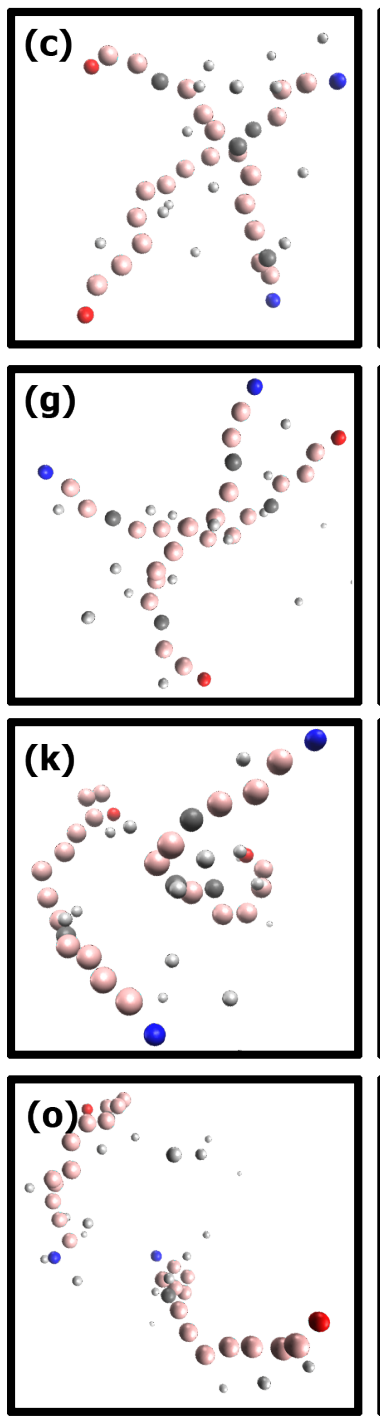

pH $-\mathrm{pKa}=4.5$
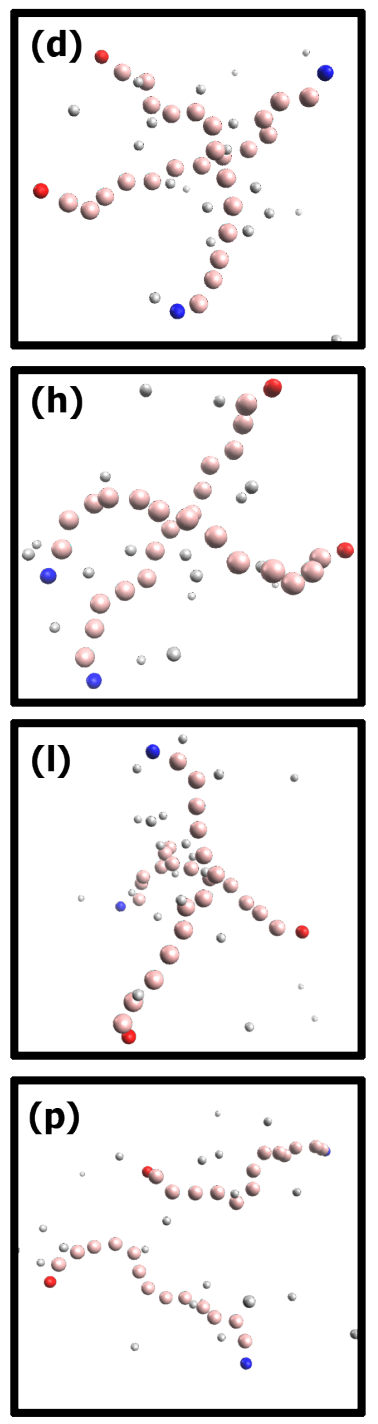

Figure S13: Trajectory snapshots for 2 semi-rigid chains and $\zeta=0$ at different $\mathrm{pH}-\mathrm{p} K_{a}$ values and $d_{\mathrm{CoMs}}$ distances. Color scheme as in Figure 6. 

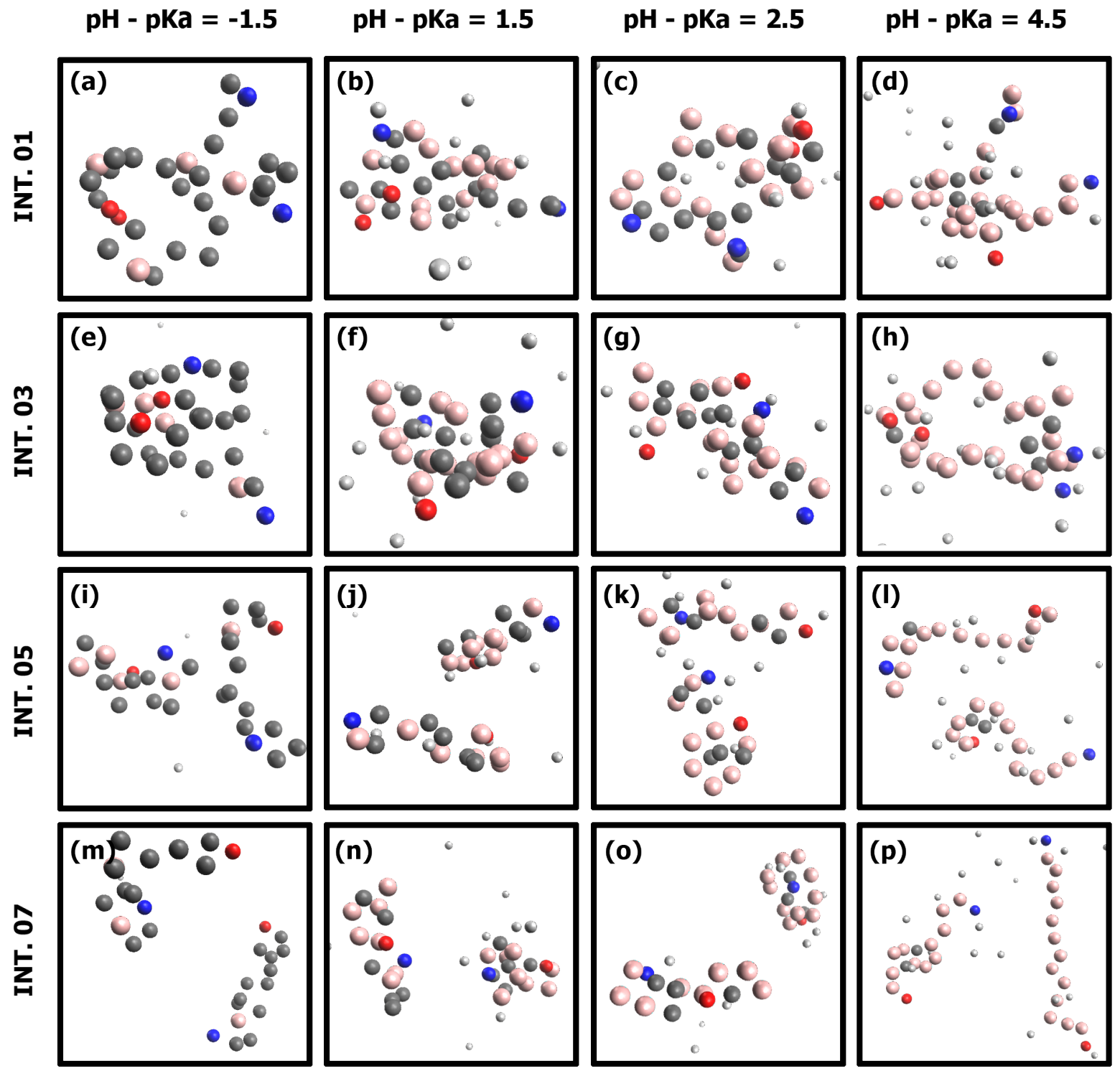

Figure S14: Trajectory snapshots for 2 flexible chains and $\zeta=2$ at different $\mathrm{pH}-\mathrm{p} K_{a}$ values and $d_{\text {CoMs }}$ distances. Color scheme as in Figure 6. 


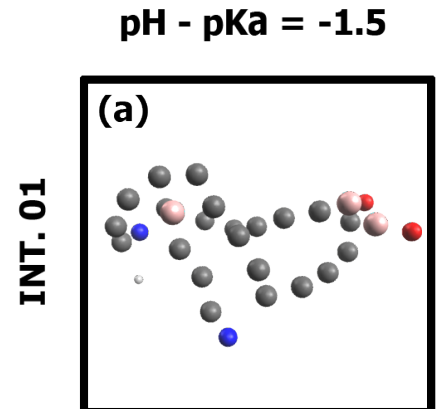

$$
\text { pH }- \text { pKa }=1.5
$$

pH - pKa $=2.5$

pH - pKa $=4.5$
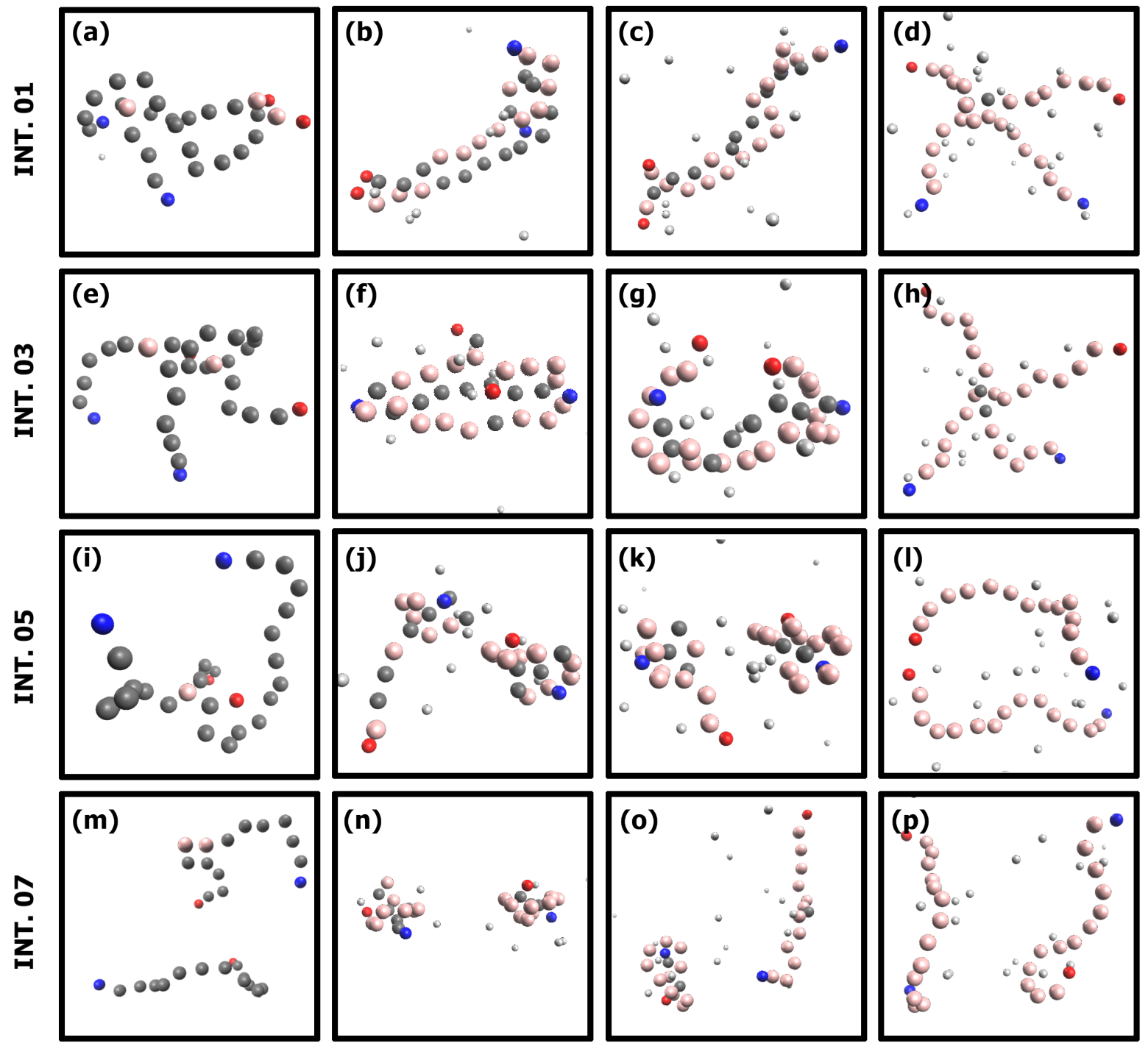

Figure S15: Trajectory snapshots for 2 semi-rigid chainsand $\zeta=2$ at different $\mathrm{pH}-\mathrm{p} K_{a}$ values and $d_{\text {CoMs }}$ distances. Color scheme as in Figure 6. 

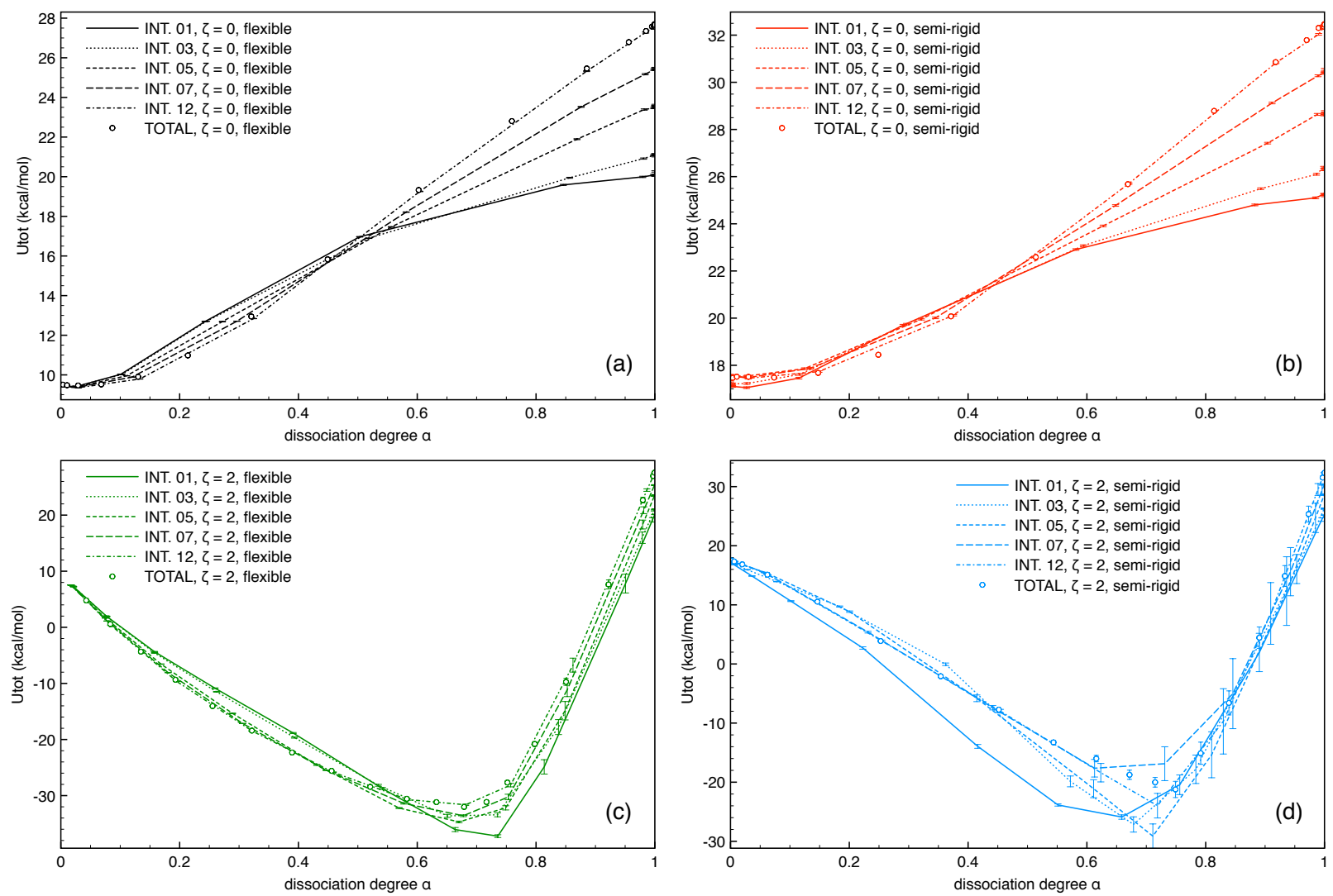

Figure S16: Total potential $U_{\text {tot }}$ (in $\mathrm{kcal} / \mathrm{mol}$ ) as function of $\alpha$ for different intervals of $d_{\text {CoMs }}$. (a) flexible, $\zeta=0$; (b) semi-rigid, $\zeta=0$; (c) flexible, $\zeta=2$; (d) semi-rigid, $\zeta=2$. 

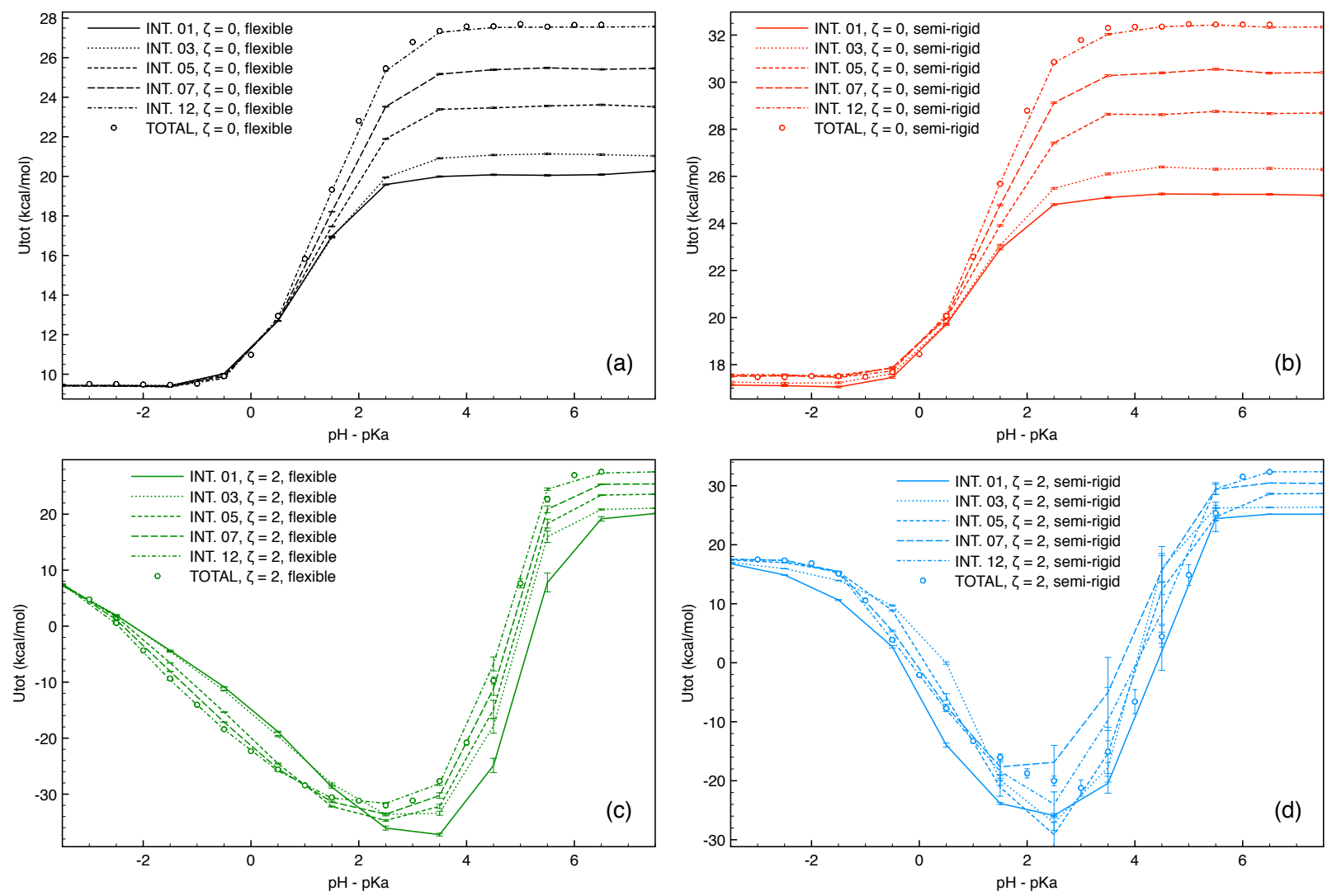

Figure S17: Total potential energy $U_{\text {tot }}$ as function of $\mathrm{pH}-\mathrm{p} K_{a}$ : (a) $\zeta=0$, flexible; (b) $\zeta=0$, semi-rigid; (c) $\zeta=2$, flexible; (d) $\zeta=2$, semi-rigid. 

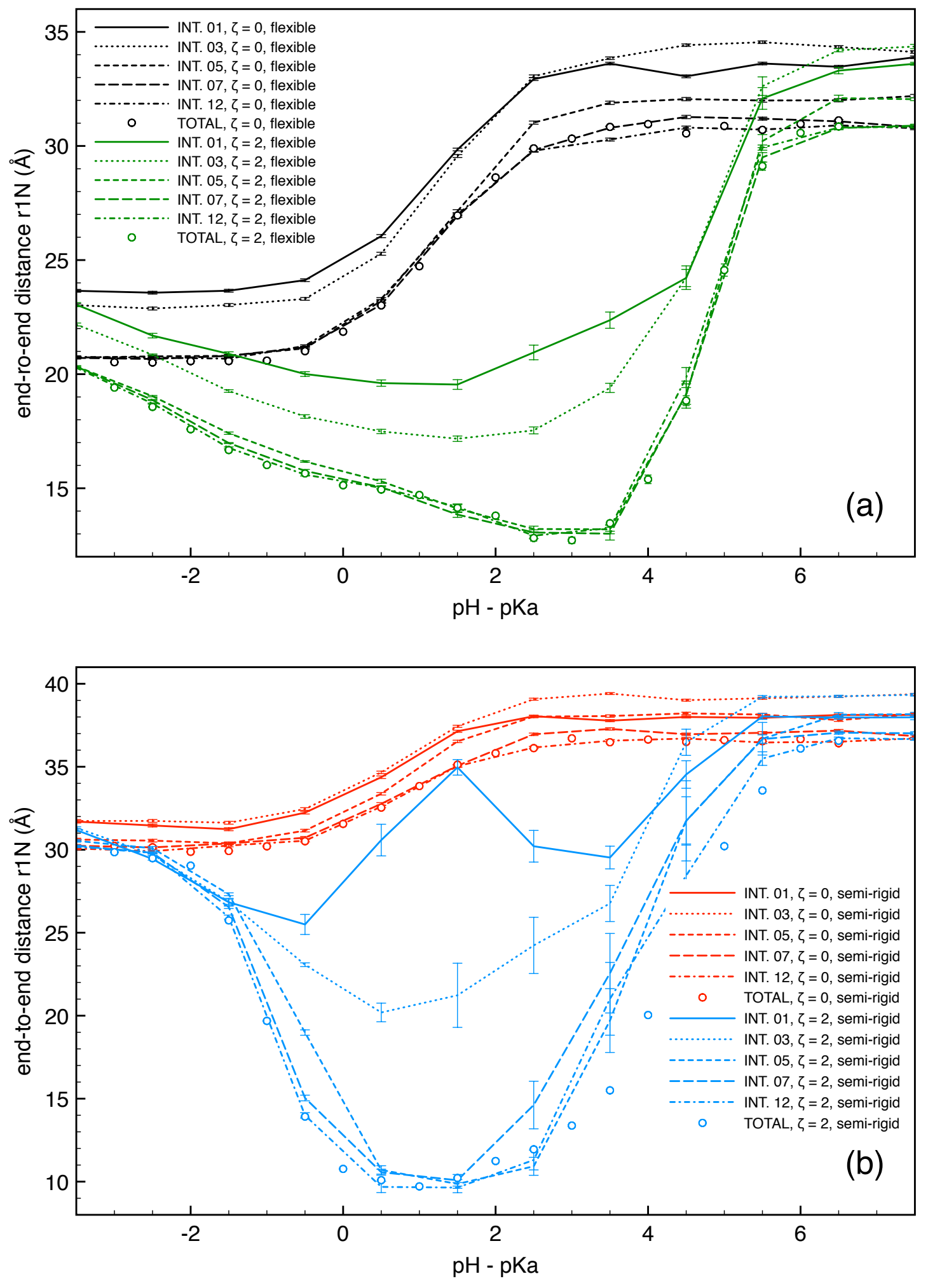

Figure S18: End-to-end distance $r_{1 \mathrm{~N}}$ as function of $\mathrm{pH}-\mathrm{p} K_{a}$. (a) flexible chains; (b) semi-rigid chains. 